\title{
Perfectly Matched Layer for Accurate FDTD for Anisotropic Magnetized Plasma
}

\author{
Jeahoon Cho · Min-Seok Park · Kyung-Young Jung*
}

\begin{abstract}
In this work, we propose a stable perfectly matched layer (PML) for accurate finite-difference time-domain (FDTD) methods for analyzing electromagnetic wave propagation in the anisotropic magnetized plasma region. Toward this purpose, we apply the complex frequency-shifted PML systematically to the E-J collocated FDTD method. In specific, auxiliary PML variables are included in the matrix calculation involved in the final update equations of the E-J collocated FDTD method. Numerical examples are used to validate the proposed PML-FDTD for anisotropic magnetized plasma.
\end{abstract}

Key Words: Anisotropic Magnetized Plasma, Finite-Difference Time-Domain (FDTD) Method, Perfectly Matched Layer.

\section{INTRODUCTION}

The finite-difference time-domain (FDTD) method [1-4] has been popularly used for a variety of electromagnetic (EM) wave propagation phenomena in the Earth's atmosphere $[5,6]$. The Earth's atmosphere consists of the troposphere, the stratosphere, and the ionosphere [7]. In the troposphere and stratosphere, only the refraction and attenuation phenomena affect EM wave propagation. However, EM wave propagation in the ionosphere is complicated because of various propagation environments, such as the static magnetic field of the Earth and plasma. Therefore, it is of great importance to accurately analyze EM wave propagation in the ionosphere modeled as anisotropic magnetized plasma. Plasma is a frequency-dependent dispersive medium, and it can be implemented in FDTD using various methods, such as the recursive convolution method [8], the Ztransform method [9], and the auxiliary differential equation (ADE) method $[5,6,10]$. The ADE-FDTD method is highly preferable because it involves a simple arithmetic implementation, and it can also be straightforwardly applied to nonlinear dispersive media, unlike other methods $[11,12]$. There are two particular implementations in ADE-FDTD for the EM analysis of anisotropic magnetized plasma. First, the magnetic field $(\mathrm{H})$ and the current density $(\mathrm{J})$ are collocated at the same time step and position when discretizing J [5]; this is called the H-J collocated FDTD method. Second, the electric field (E) and J components are collocated at the same time step and position; this is called the E-J collocated FDTD method [6]. Unlike that of the H-J collocated method, the numerical stability condition of the E-J collocated method is independent of medium properties and remains the same as the Courant stability limit for free space [6]. Moreover, the E-J collocated method is more accurate than the H-J collocated method [6]. Note that unconditionally stable FDTDs for magnetized plasma have been proposed based on the weighted Laguerre polynomials [13] or the CrankNicolson-approximate-decoupling algorithm [14].

Manuscript received March 16, 2020 ; Revised June 27, 2020 ; Accepted July 27, 2020. (ID No. 20200316-035J)

Department of Electronics and Computer Engineering, Hanyang University, Seoul, Korea.

"Corresponding Author: Kyung-Young Jung (e-mail: kyjung3@hanyang.ac.kr)

This is an Open-Access article distributed under the terms of the Creative Commons Attribution Non-Commercial License (http://creativecommons.org/licenses/by-nc/4.0) which permits unrestricted non-commercial use, distribution, and reproduction in any medium, provided the original work is properly cited.

(c) Copyright The Korean Institute of Electromagnetic Engineering and Science. All Rights Reserved. 
In FDTD, applying an appropriate boundary condition $(\mathrm{ABC})$ to truncate the computational domain is necessary. Currently, the most efficient and commonly used $\mathrm{ABC}$ for FDTD is the perfectly matched layer (PML) $[15,16]$. However, the PML implementation is not straightforward in the E-J collocated method, as a matrix calculation is involved in the FDTD update equations. To the best of our knowledge, the PML implementation to the E-J collocated FDTD scheme for anisotropic magnetized plasma has not yet been discussed. In this work, we propose a stable PML implementation suitable for the E-J collocated FDTD approach for anisotropic magnetized plasma. Toward this purpose, we employ complex stretching variables for the nabla operator when deriving FDTD update equations. Numerical examples are used to validate our proposed PML implementation.

\section{FORMULATION}

\section{FDTD Update Equations}

Let us consider the FDTD update equations for anisotropic magnetized plasma based on the E-J collocated method. The EM wave propagation in the anisotropic magnetized plasma region can be analyzed by Maxwell's equations coupled with the Lorentz equation of motion. The governing equation set is given by

$$
\begin{gathered}
\nabla \times \mathbf{H}=\varepsilon_{0} \frac{\partial \mathbf{E}}{\partial t}+\mathbf{J} \\
\nabla \times \mathbf{E}=-\mu_{0} \frac{\partial \mathbf{H}}{\partial t} \\
\frac{\partial \mathbf{J}}{\partial t}+v_{c} \mathbf{J}=\varepsilon_{0} \omega_{p}^{2} \mathbf{E}+\boldsymbol{\omega}_{b} \times \mathbf{J},
\end{gathered}
$$

where $v_{c}$ is the collision frequency, $\omega_{p}$ is the plasma frequency, $\boldsymbol{\omega}_{b}$ is the cyclotron frequency, and $\varepsilon_{0}$ and $\mu_{0}$ are the permittivity and permeability of free space, respectively. The cyclotron frequency is a function of the static magnetic field. Therefore, the crossproduct term in Eq. (3) can lead to anisotropy of plasma so that the EM wave behavior depends on the direction of the static magnetic field relative to the EM wave propagation direction. In the E-J collocated method, the current density vectors are collocated at the same time step and position of the electric field vectors. Therefore, by applying the central difference scheme (CDS) in both time and space to Eqs. (1)-(3), one can obtain the FDTD update equations consisting of three standard update equations for the magnetic field components and six coupled update equations for the electric field and current density components.

The update equations for Eq. (1) using CDS are as follows:

$$
\begin{aligned}
& \left.H_{x}\right|_{i, j+1 / 2, k+1 / 2} ^{n+1 / 2}=\left.H_{x}\right|_{i, j+j+1 / 2, k+1 / 2} ^{n-1 / 2}+\frac{\Delta t}{\mu_{0} \Delta z}\left(E_{\left.y\right|_{i, j+1 / 2, k+1} ^{n}}^{n}-\left.E_{y}\right|_{i, j+1 / 2, k} ^{n}\right) \\
& -\frac{\Delta t}{\mu_{0} \Delta y}\left(E_{z l_{i, j+1, k+1 / 2}^{n}}^{n}-E_{z z_{i, j, k+1 / 2}^{n}}^{n}\right) \\
& \left.H_{y}\right|_{i+1 / 2, j, k+1 / 2} ^{n+1 / 2}=\left.H_{y}\right|_{i+1 / 2, j, j+1 / 2} ^{n-1 / 2}+\frac{\Delta t}{\mu_{0} \Delta x}\left(E_{\left.z\right|_{i+1, j, k+1 / 2} ^{n}}^{n}-\left.E_{z}\right|_{i, j, j+k+1 / 2} ^{n}\right) \\
& -\frac{\Delta t}{\mu_{0} \Delta z}\left(\left.E_{x}\right|_{i+1 / 2, j, k+1} ^{n}-\left.E_{x}^{\mid n}\right|_{i+1 / 2, j, k} ^{n}\right) \\
& \left.H_{z}\right|_{i+1 / 2, j+1 / 2, k} ^{n+1 / 2}=H_{z} z_{i+1 / 2, j+1 / 2, k}^{n-1 / 2}+\frac{\Delta t}{\mu_{0} \Delta y}\left(\left.E_{x}\right|_{i+1 / 2, j+1, k} ^{n}-\left.E_{x}\right|_{i+1 / 2, j, k} ^{n}\right) \\
& -\frac{\Delta t}{\mu_{0} \Delta x}\left(\left.E_{y}\right|_{i+1, j+1+2, k} ^{n}-\left.E_{y}\right|_{i, j+1 / 2, k} ^{n}\right) \text {. }
\end{aligned}
$$

The update equations for Eq. (2) using CDS are as follows:

$$
\begin{aligned}
& \left.E_{x}\right|_{i+1 / 2, j, k} ^{n+1}+\left.\frac{\Delta t}{2 \varepsilon_{0}} J_{x}\right|_{i+1 / 2, j, k} ^{n+1}=\left.E_{x}\right|_{i+1 / 2, j, k} ^{n}-\left.\frac{\Delta t}{2 \varepsilon_{0}} J_{x}\right|_{i+1 / 2, j, k} ^{n} \\
& +\frac{\Delta t}{\varepsilon_{0} \Delta y}\left(H_{z} z_{i+1 / 2, j+1 / 2, k}^{n+1 / 2}-H_{z} z_{i+1 / 2, j-1 / 2, k}^{n+1 / 2}\right) \\
& -\frac{\Delta t}{\varepsilon_{0} \Delta z}\left(\left.H_{y}\right|_{i+1 / 2, j, k+1 / 2} ^{n+1 / 2}-\left.H_{y}\right|_{i+1 / 2, j, k+1 / 2} ^{n+1 / 2}\right) \\
& \left.E_{y}\right|_{i, j+1 / 2, k} ^{n+1}+\left.\frac{\Delta t}{2 \varepsilon_{0}} J_{y}\right|_{i, j+1 / 2, k} ^{n+1}=\left.E_{y}\right|_{i, j+1 / 2, k} ^{n}-\left.\frac{\Delta t}{2 \varepsilon_{0}} J_{y}\right|_{i, j+1 / 2, k} ^{n} \\
& +\frac{\Delta t}{\varepsilon_{0} \Delta z}\left(H_{x}{ }_{i, j+1+1 / 2, k+1 / 2}^{n+1 / 2}-H_{x} x_{i, j+1 / 2, k-1 / 2}^{n+1 / 2}\right) \\
& -\frac{\Delta t}{\varepsilon_{0} \Delta x}\left(H_{z}{ }_{i+1 / 2, j+1 / 2, k}^{n+1 / 2}-H_{z} z_{i-1 / 2, j+1 / 2, k}^{n+1 / 2}\right)
\end{aligned}
$$

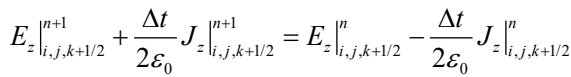

$$
\begin{aligned}
& +\frac{\Delta t}{\varepsilon_{0} \Delta x}\left(H_{y}{ }_{i+1 / 2, j, k+1 / 2}^{n+1 / 2}-H_{y} y_{i-1 / 2, j, k+1 / 2}^{n+1 / 2}\right) \\
& -\frac{\Delta t}{\varepsilon_{0} \Delta y}\left(H_{x} x_{i, j+1 / 2, k+1 / 2}^{n+1 / 2}-\left.H_{x}\right|_{i, j-1 / 2, k+1 / 2} ^{n+1 / 2}\right) .
\end{aligned}
$$

The update equations for Eq. (3) using CDS are as follows:

$$
\begin{aligned}
& \left(1+\frac{v_{c} \Delta t}{2}\right) J_{x}^{n+1+1 / 2, j, k}{ }^{n+1}+\left.\frac{\omega_{b z} \Delta t}{2} J_{y}\right|_{i, j+1 / 2, k} ^{n+1}-\frac{\omega_{b y} \Delta t}{2} J_{z} z_{i, j, k+1 / 2}^{n+1} \\
& -\left.\frac{\varepsilon_{0} \omega_{p}^{2} \Delta t}{2} E_{x}\right|_{i+1 / 2, j, k} ^{n+1}=\left.\frac{\varepsilon_{0} \omega_{p}^{2} \Delta t}{2} E_{x}\right|_{i+1 / 2, j, k} ^{n} \\
& +\left.\left(1-\frac{v_{c} \Delta t}{2}\right) J_{x}\right|_{i+1 / 2, j, k} ^{n}-\left.\frac{\omega_{b z} \Delta t}{2} J_{y}\right|_{i, j+1 / 2, k} ^{n}+\left.\frac{\omega_{b y} \Delta t}{2} J_{z}\right|_{i, j, k+1 / 2} ^{n} \\
& -\left.\frac{\omega_{b z} \Delta t}{2} J_{x}^{n+1}\right|_{i+1 / 2, j, k} ^{n+1}+\left.\left(1+\frac{v_{c} \Delta t}{2}\right) J_{y}\right|_{i, j+1 / 2, k} ^{n+1}+\left.\frac{\omega_{b x} \Delta t}{2} J_{z}\right|_{i, j, k+1 / 2} ^{n+1} \\
& -\left.\frac{\varepsilon_{0} \omega_{p}^{2} \Delta t}{2} E_{y}\right|_{i, j+1 / 2, k} ^{n+1}=\left.\frac{\varepsilon_{0} \omega_{p}^{2} \Delta t}{2} E_{y}\right|_{i, j+1 / 2, k} ^{n} \\
& +\left.\frac{\omega_{b z} \Delta t}{2} J_{x}\right|_{i+1 / 2, j, k} ^{n}+\left.\left(1-\frac{v_{c} \Delta t}{2}\right) J_{y}\right|_{i, j+1 / 2, k} ^{n}-\left.\frac{\omega_{b x} \Delta t}{2} J_{z}\right|_{i, j, k+1 / 2} ^{n} \\
& \left.\frac{\omega_{b y} \Delta t}{2} J_{x}\right|_{i+1 / 2, j, k} ^{n+1}-\left.\frac{\omega_{b x} \Delta t}{2} J_{y}\right|_{i, j+1 / 2, k} ^{n+1}+\left(1+\frac{v_{c} \Delta t}{2}\right) J_{z} z_{i, j, k+1 / 2}^{n+1} \\
& -\frac{\varepsilon_{0} \omega_{p}^{2} \Delta t}{2} E_{z i, j, k+1 / 2}^{n+1}=\left.\frac{\varepsilon_{0} \omega_{p}^{2} \Delta t}{2} E_{z}^{n}\right|_{i, j, k+1 / 2} ^{n} \\
& -\left.\frac{\omega_{b y} \Delta t}{2} J_{x}\right|_{i+1 / 2, j, k} ^{n}+\left.\frac{\omega_{b x} \Delta t}{2} J_{y}\right|_{i, j+1 / 2, k} ^{n}+\left.\left(1-\frac{v_{c} \Delta t}{2}\right) J_{z}\right|_{i, j, k+1 / 2} ^{n} .
\end{aligned}
$$


Here, the superscript and the subscript refer to the time and spatial indexing, respectively. The $\omega_{b x}, \omega_{b y}$, and $\omega_{b z}$ are the cyclotron frequencies along each direction. As shown in Eqs. (7)-(12), the update equations of $\mathrm{E}$ and $\mathrm{J}$ are required at the same time step, and thus the final FDTD update equations are expressed in a matrix form. For brevity's sake, we use the following notation:

$$
\begin{gathered}
\left.H_{x}\right|_{i, j+1 / 2, k+1 / 2} ^{n+1 / 2}-\left.H_{x}\right|_{i, j+1 / 2, k-1 / 2} ^{n+1 / 2}=\left.\partial H_{x}\right|_{\hat{k}} ^{n+1 / 2}, \\
\left.H_{y}\right|_{i+1 / 2, j, k+1 / 2} ^{n+1 / 2}-\left.H_{y}\right|_{i-1 / 2, j, k+1 / 2} ^{n+1 / 2}=\left.\partial H_{y}\right|_{i} ^{n+1 / 2}, \\
\left.H_{z}\right|_{i+1 / 2, j+1 / 2, k} ^{n+1 / 2}-\left.H_{z}\right|_{i+1 / 2, j-1 / 2, k} ^{n+1 / 2}=\left.\partial H_{z}\right|_{j} ^{n+1 / 2} \\
{\left[\begin{array}{l}
\left.E_{x}\right|_{i+1 / 2, j, k} ^{n+1} \\
\left.E_{y}\right|_{i, j+1 / 2, k} ^{n+1} \\
\left.E_{z}\right|_{i, j, k+1 / 2} ^{n+1} \\
\left.J_{x}\right|_{i+1 / 2, j, k} ^{n+1} \\
\left.J_{y}\right|_{i, j+1 / 2, k} ^{n+1} \\
\left.J_{z}\right|_{i, j, k+1 / 2} ^{n+1}
\end{array}\right]=\left[\begin{array}{l}
\left.E_{x}\right|^{n} \\
\left.\left.E_{y}\right|^{n-1} \mathbf{B}\right]\left[\left.\begin{array}{l}
\left.\partial H_{z}\right|_{j} ^{n+1 / 2} \\
\left.E_{z}\right|^{n} \\
\left.J_{x}\right|^{n} \\
\left.J_{y}\right|^{n} \\
\left.J_{z}\right|^{n}
\end{array}\right|_{\hat{k}} ^{n+1 / 2}\right. \\
\left.\partial H_{x}\right|_{\hat{k}} ^{n+1 / 2} \\
\left.\partial H_{z}\right|_{i} ^{n+1 / 2} \\
\left.\partial H_{y}\right|_{i} ^{n+1 / 2} \\
\left.\partial H_{x}\right|_{j} ^{n+1 / 2}
\end{array}\right]+\left[\mathbf{A}^{-1}\right.}
\end{gathered}
$$

$$
\mathbf{A}=\left[\begin{array}{cccccc}
1 & 0 & 0 & \frac{\Delta t}{2 \varepsilon_{0}} & 0 & 0 \\
0 & 1 & 0 & 0 & \frac{\Delta t}{2 \varepsilon_{0}} & 0 \\
0 & 0 & 1 & 0 & 0 & \frac{\Delta t}{2 \varepsilon_{0}} \\
-\frac{\varepsilon_{0} \omega_{p}^{2} \Delta t}{2} & 0 & 0 & 1+\frac{v_{c} \Delta t}{2} & \frac{\omega_{b z} \Delta t}{2} & -\frac{\omega_{b y} \Delta t}{2} \\
0 & -\frac{\varepsilon_{0} \omega_{p}^{2} \Delta t}{2} & 0 & -\frac{\omega_{b z} \Delta t}{2} & 1+\frac{v_{c} \Delta t}{2} & \frac{\omega_{b x} \Delta t}{2} \\
0 & 0 & -\frac{\varepsilon_{0} \omega_{p}^{2} \Delta t}{2} & \frac{\omega_{b y} \Delta t}{2} & -\frac{\omega_{b x} \Delta t}{2} & 1+\frac{v_{c} \Delta t}{2}
\end{array}\right]
$$

$$
\begin{gathered}
\mathbf{B}=\left[\begin{array}{cccccc}
1 & 0 & 0 & -\frac{\Delta t}{2 \varepsilon_{0}} & 0 & 0 \\
0 & 1 & 0 & 0 & -\frac{\Delta t}{2 \varepsilon_{0}} & 0 \\
0 & 0 & 1 & 0 & 0 & -\frac{\Delta t}{2 \varepsilon_{0}} \\
\frac{\varepsilon_{0} \omega_{p}^{2} \Delta t}{2} & 0 & 0 & 1-\frac{v_{c} \Delta t}{2} & -\frac{\omega_{b z} \Delta t}{2} & \frac{\omega_{b y} \Delta t}{2} \\
0 & \frac{\varepsilon_{0} \omega_{p}^{2} \Delta t}{2} & 0 & \frac{\omega_{b b} \Delta t}{2} & 1-\frac{v_{c} \Delta t}{2} & -\frac{\omega_{b x} \Delta t}{2} \\
0 & 0 & \frac{\varepsilon_{0} \omega_{p}^{2} \Delta t}{2} & -\frac{\omega_{b y} \Delta t}{2} & \frac{\omega_{b x} \Delta t}{2} & 1-\frac{v_{c} \Delta t}{2}
\end{array}\right] \\
\mathbf{C}=\left[\begin{array}{cccccc}
\frac{\Delta t}{\varepsilon_{0} \Delta y} & -\frac{\Delta t}{\varepsilon_{0} \Delta z} & 0 & 0 & 0 & 0 \\
0 & 0 & \frac{\Delta t}{\varepsilon_{0} \Delta z} & -\frac{\Delta t}{\varepsilon_{0} \Delta x} & 0 & 0 \\
0 & 0 & 0 & 0 & \frac{\Delta t}{\varepsilon_{0} \Delta x} & -\frac{\Delta t}{\varepsilon_{0} \Delta y} \\
0 & 0 & 0 & 0 & 0 & 0 \\
0 & 0 & 0 & 0 & 0 & 0 \\
0 & 0 & 0 & 0 & 0 & 0
\end{array}\right],
\end{gathered}
$$

where $\mathrm{A}[6 \times 6], \mathrm{B}[6 \times 6]$, and $\mathrm{C}[6 \times 6]$ are the coefficient marices that depend on the anisotropic magnetized plasma properties and the FDTD modeling parameters for time and space. It should be emphasized that the matrix calculation is performed only once before FDTD time marching, and thus the demanding computational cost is negligible. Note that there is a spatially non-collocated status of electric fields, magnetic fields, and current densities. To address this problem, we use the space averaging technique for all the spatially non-collocated components to maintain second-order accuracy [6]. For example, the final update equation of $E_{x}$ for anisotropic magnetized plasma can be expressed as

$$
\begin{aligned}
& \left.E_{x}^{n+1}\right|_{i+1 / 2, j, k} ^{n}=\left.\left[\mathbf{A}^{-1} \mathbf{B}\right]^{\{1,1\}} E_{x}\right|_{i+1 / 2, j, k} ^{n} \\
& +\left[\mathbf{A}^{-1} \mathbf{B}\right]^{(1,2\rangle}\left(\left.\tilde{E}_{y}\right|^{n}\right)_{i+1 / 2, j, k}+\left[\mathbf{A}^{-1} \mathbf{B}\right]^{(1,3)}\left(\left.\tilde{E}_{z}\right|^{n}\right)_{i+1 / 2, j, k} \\
& +\left.\left[\mathbf{A}^{-1} \mathbf{B}\right]^{(1,4)} J_{x}\right|_{i+1 / 2, j, k} ^{n} \\
& +\left[\mathbf{A}^{-1} \mathbf{B}\right]^{\langle 1,5)}\left(\left.\tilde{J}_{y}\right|^{n}\right)_{i+1 / 2, j, k}+\left[\mathbf{A}^{-1} \mathbf{B}\right]^{\langle 1,6\rangle}\left(\left.\tilde{J}_{z}\right|^{n}\right)_{i+1 / 2, j, k} \\
& +\left[\mathbf{A}^{-1} \mathbf{C}\right]^{(1,1)}\left(\left.\partial H_{z}\right|_{j} ^{n+1 / 2}\right)+\left[\mathbf{A}^{-1} \mathbf{C}\right]^{(1,2)}\left(\left.\partial H_{y}\right|_{\bar{k}} ^{n+1 / 2}\right) \\
& +\left[\mathbf{A}^{-1} \mathbf{C}\right]^{\{1,3\rangle}\left(\left.\partial \tilde{H}_{x}\right|_{\tilde{k}} ^{n+1 / 2}\right)_{i+1 / 2, j, k}+\left[\mathbf{A}^{-1} \mathbf{C}\right]^{(1,4)}\left(\left.\partial \tilde{H}_{z}\right|_{\tilde{i}} ^{n+1 / 2}\right)_{i+1 / 2, j, k} \\
& +\left[\mathbf{A}^{-1} \mathbf{C}\right]^{(1,5\rangle}\left(\left.\partial \tilde{H}_{y}\right|_{i} ^{n+1 / 2}\right)_{i+1 / 2, j, k}+\left[\mathbf{A}^{-1} \mathbf{C}\right]^{(1,6)}\left(\left.\partial \tilde{H}_{x}\right|_{j} ^{n+1 / 2}\right)_{i+1 / 2, j, k},
\end{aligned}
$$

where the superscript $\langle a, b\rangle$ refers to the corresponding index of the matrix element of $\left[\mathrm{A}^{-1} \mathrm{~B}\right]$ and $\left[\mathrm{A}^{-1} \mathrm{C}\right]$. In Eq. (17), the electric fields $(\tilde{E})$, magnetic fields $(\widetilde{H})$, and current densities $(\tilde{J})$ should be considered by applying spatial averaging at position $(i$ $+1 / 2, j, k)$ of the $E_{x}$ field component. The other coupled components, $E_{y}, E_{z}, J_{x}, J_{y}$, and $J_{z}$, are obtained in a similar manner as Eq. (17). Note that the final update equations for the magnetic field component can be obtained through the standard FDTD process (Eqs. (4)-(6)) because there is no field coupled with other field components.

\section{PML-FDTD Update Equations}

Let us consider the ABC for the E-J collocated FDTD method. In this work, the complex frequency-shifted (CFS)PML is employed to prevent the spurious late-time growth of EM fields [16]. By using a modified nabla operator with complex stretching variables,

$$
\nabla \rightarrow \tilde{\nabla}=\hat{x} \frac{1}{s_{x}} \frac{\partial}{\partial x}+\hat{y} \frac{1}{s_{y}} \frac{\partial}{\partial y}+\hat{z} \frac{1}{s_{z}} \frac{\partial}{\partial z},
$$

where a CFS stretching is utilized so that

$$
s_{\zeta}=\kappa_{\zeta}+\frac{\sigma_{\zeta}}{\alpha_{\zeta}+j \omega \varepsilon_{0}} .
$$

Here, $\zeta=x, y, z$. The CFS stretching $\left(\alpha_{\zeta} \neq 0\right)$ leads to a slightly more costly time-domain implementation than the standard PML stretching $\left(\alpha_{\zeta}=0\right)$, but the former is more effective at absorbing evanescent waves and low-frequency fields, as it can correctly operate for $\omega \rightarrow 0$. Except for considering $\kappa_{\zeta}$ variable, the update equations of the CFS-PML E-J collocated FDTD 
are almost the same as those of the E-J collocated FDTD based on Eqs. (7)-(12). In general, PML implementation is straightforwardly applied to most FDTD update equations. However, it is not straightforward in the E-J collocated FDTD, as the current density components are collocated at the same time step and position of the electric field components. Therefore, the CFS-PML implementation is also included in matrix form for the update equations of the E-J collocated FDTD:

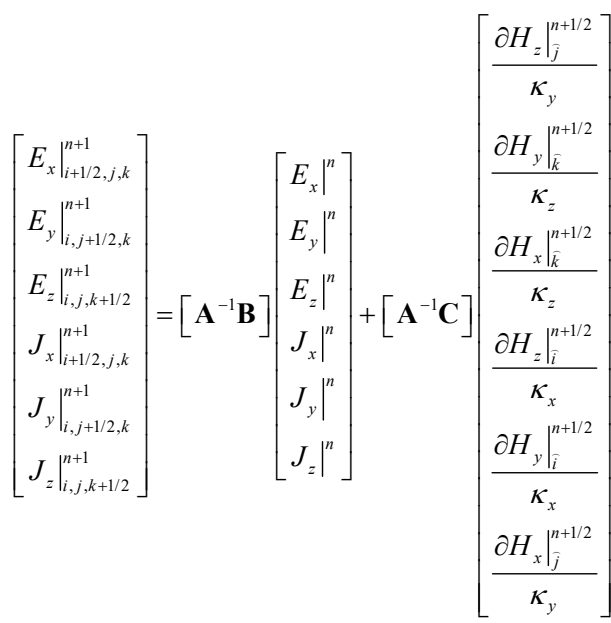

$$
\begin{aligned}
& +\left[\mathbf{A}^{-1} \mathbf{D}\right]\left[\begin{array}{c}
\left.f_{x y}\right|^{n+1 / 2} \\
\left.f_{x z}\right|^{n+1 / 2} \\
\left.f_{y z}\right|^{n+1 / 2} \\
\left.f_{y x}\right|^{n+1 / 2} \\
\left.f_{z x}\right|^{n+1 / 2} \\
\left.f_{z y}\right|^{n+1 / 2}
\end{array}\right] \\
& \mathbf{D}=\left[\begin{array}{cccccc}
\frac{\Delta t}{\varepsilon_{0}} & -\frac{\Delta t}{\varepsilon_{0}} & 0 & 0 & 0 & 0 \\
0 & 0 & \frac{\Delta t}{\varepsilon_{0}} & -\frac{\Delta t}{\varepsilon_{0}} & 0 & 0 \\
0 & 0 & 0 & 0 & \frac{\Delta t}{\varepsilon_{0}} & -\frac{\Delta t}{\varepsilon_{0}} \\
0 & 0 & 0 & 0 & 0 & 0 \\
0 & 0 & 0 & 0 & 0 & 0 \\
0 & 0 & 0 & 0 & 0 & 0
\end{array}\right]
\end{aligned}
$$

The final update equation of $E_{x}$ for anisotropic magnetized plasma in the PML region can be expressed as

$$
\begin{aligned}
& \left.E_{x}\right|_{i+1 / 2, j, k} ^{n+1}=\left.\left[\mathbf{A}^{-1} \mathbf{B}\right]^{\langle 1,1\rangle} E_{x}\right|_{i+1 / 2, j, k} ^{n} \\
& +\left[\mathbf{A}^{-1} \mathbf{B}\right]^{\langle 1,2\rangle}\left(\left.\tilde{E}_{y}\right|^{n}\right)_{i+1 / 2, j, k}+\left[\mathbf{A}^{-1} \mathbf{B}\right]^{\langle 1,3\rangle}\left(\left.\tilde{E}_{z}\right|^{n}\right)_{i+1 / 2, j, k} \\
& +\left.\left[\mathbf{A}^{-1} \mathbf{B}\right]^{(1,4\rangle} J_{x}\right|_{i+1 / 2, j, k} ^{n} \\
& +\left[\mathbf{A}^{-1} \mathbf{B}\right]^{\{1,5\rangle}\left(\left.\tilde{J}_{y}\right|^{n}\right)_{i+1 / 2, j, k}+\left[\mathbf{A}^{-1} \mathbf{B}\right]^{\langle 1,6\rangle}\left(\left.\tilde{J}_{z}\right|^{n}\right)_{i+1 / 2, j, k} \\
& +\left[\mathbf{A}^{-1} \mathbf{C}\right]^{(1,1)}\left(\frac{\left.\partial H_{z}\right|_{j} ^{n+1 / 2}}{\kappa_{y}}\right)+\left[\mathbf{A}^{-1} \mathbf{C}\right]^{\langle 1,2\rangle}\left(\frac{\left.\partial H_{y}\right|_{\hat{k}} ^{n+1 / 2}}{\kappa_{z}}\right)
\end{aligned}
$$

$$
\begin{aligned}
& +\left[\mathbf{A}^{-1} \mathbf{C}\right]^{(1,3,3}\left(\frac{\partial \tilde{H}_{x} \kappa_{\tilde{k}}^{(n+1 / 2}}{\kappa_{z}}\right)_{i+1 / 2, j, k}+\left[\mathbf{A}^{-1} \mathbf{C}\right]^{(1,4)}\left(\frac{\partial \tilde{H}_{z} z_{\tilde{i}}^{n+1 / 2}}{\kappa_{x}}\right)_{i+1 / 2, j, k} \\
& +\left[\mathbf{A}^{-1} \mathbf{C}\right]^{(1,5)}\left(\frac{\left.\partial \tilde{H}_{y}\right|_{i} ^{n+1 / 2}}{\kappa_{x}}\right)_{i+1 / 2, j, k}+\left[\mathbf{A}^{-1} \mathbf{C}\right]^{(1,6)}\left(\frac{\left.\partial \tilde{H}_{x}\right|_{j} ^{n+1 / 2}}{\kappa_{y}}\right)_{i+1 / 2, j, k} \\
& +\left.\left[\mathbf{A}^{-1} \mathbf{D}\right]^{(1,1\rangle} f_{x x}\right|_{i+1 / 2, j, j, k} ^{n+1 / 2}+\left[\mathbf{A}^{-1} \mathbf{D}\right]^{(1,2\rangle} f_{x z}^{n+1+1 / 2, j, j, k} \\
& +\left[\mathbf{A}^{-1} \mathbf{D}\right]^{(1,3)}\left(\left.\tilde{f}_{y z}\right|^{n+1 / 2}\right)_{i+1 / 2, j, k}+\left[\mathbf{A}^{-1} \mathbf{D}\right]^{\langle 1,4)}\left(\left.\tilde{f}_{y x}\right|^{n+1 / 2}\right)_{i+1 / 2, j, k} \\
& +\left[\mathbf{A}^{-1} \mathbf{D}\right]^{(1,5\rangle}\left(\left.\tilde{f}_{z x}\right|^{n+1 / 2}\right)_{i+1 / 2, j, k}+\left[\mathbf{A}^{-1} \mathbf{D}\right]^{\langle 1,6)}\left(\left.\tilde{f}_{z y}\right|^{n+1 / 2}\right)_{i+1 / 2, j, k} .
\end{aligned}
$$

Here, $f_{x y}, f_{x z}, f_{y z}, f_{y x}, f_{z x}$, and $f_{z y}$ are the auxiliary variables of CFS-PML implementations [17]. The auxiliary variables are given by

$$
\begin{aligned}
& \left.f_{x y}\right|_{i+1 / 2, j, j, k} ^{n+1 / 2}=\left.b_{y} f_{x y}\right|_{i+1 / 2, j, k} ^{n-1 / 2}+a_{y} \frac{\left.\partial H_{z}\right|_{j} ^{n+1 / 2}}{\Delta y} \\
& \left.f_{x z}\right|_{i+1 / 2, j, k} ^{n+1 / 2}=\left.b_{z} f_{x x}\right|_{i+1 / 2, j, k} ^{n-1 / 2}+a_{z} \frac{\left.\partial H_{y}\right|_{k} ^{n+1 / 2}}{\Delta z} \\
& a_{\zeta}=\frac{\sigma_{\zeta}}{\left(\sigma_{\zeta} \kappa_{\zeta}+\kappa_{\zeta}^{2} \alpha_{\zeta}\right)}\left(e^{-\left(\frac{\sigma_{\zeta}}{\kappa_{\zeta}}+\alpha_{\zeta}\right) \frac{\Delta t}{\varepsilon_{0}}}-1\right) \\
& b_{\zeta}=e^{-\left(\frac{\sigma_{\zeta}}{\kappa_{\zeta}}+\alpha_{\zeta}\right) \frac{\Delta t}{\varepsilon_{0}}}, \quad(\zeta=x, y, \text { or } z)
\end{aligned}
$$

and $\sigma_{\zeta}, \kappa_{\zeta}$, and $\alpha_{\zeta}$ are the parameters related to the CFS-PML [16]:

$$
\begin{gathered}
\sigma_{\zeta}=\sigma_{\zeta, \text { max }}\left(\frac{\zeta}{d}\right)^{m} \\
\kappa_{\zeta}=1+\left(\kappa_{\zeta, \text { max }}-1\right)\left(\frac{\zeta}{d}\right)^{m} \\
\alpha_{\zeta}=\alpha_{\zeta, \text { max }}\left[\frac{d-\zeta}{d}\right]
\end{gathered}
$$

where $d$ is the thickness of the PML. The remaining auxiliary variables can be obtained similarly to Eq. (23) and Eq. (24). $\mathrm{D}[6 \times 6]$ is the coefficient matrix that depends on the modeling parameters. Similarly, the PML implementation should be considered for the other coupled components $\left(E_{y}, E_{z}, J_{x}, J_{y}\right.$, and $\left.J_{z}\right)$ in the matrix of Eq. (20).

Before proceeding with the numerical examples, note that other PML implementation can be possible. Similar to conventional PML implementation, PML can be implemented by simply adding auxiliary variables. In this approach, the final update equation of $E_{x}$ for anisotropic magnetized plasma in the PML region is expressed as

$$
\begin{aligned}
& E_{x} x_{i+1 / 2, j, k}^{n+1}=\left.\left[\mathbf{A}^{-1} \mathbf{B}\right]^{(1,1)} E_{x}^{\mid n}\right|_{i+1 / 2, j, k} ^{n} \\
& +\left[\mathbf{A}^{-1} \mathbf{B}\right]^{(1,2)}\left(\left.\tilde{E}_{y}\right|^{n}\right)_{i+1 / 2, j, k}+\left[\mathbf{A}^{-1} \mathbf{B}\right]^{(1,3)}\left(\left.\tilde{E}_{z}\right|^{n}\right)_{i+1 / 2, j, k}
\end{aligned}
$$




$$
\begin{aligned}
& +\left.\left[\mathbf{A}^{-1} \mathbf{B}\right]^{(1,4)} J_{x}\right|_{i+1 / 2, j, k} ^{n} \\
& +\left[\mathbf{A}^{-1} \mathbf{B}\right]^{1,5\rangle}\left(\left.\tilde{J}_{y}\right|^{n}\right)_{i+1 / 2, j, k}+\left[\mathbf{A}^{-1} \mathbf{B}\right]^{(1,6)}\left(\left.\tilde{J}_{z}\right|^{n}\right)_{i+1 / 2, j, k} \\
& +\left[\mathbf{A}^{-1} \mathbf{C}\right]^{(1,1)}\left(\frac{\left.\partial H_{z}\right|_{j} ^{n+1 / 2}}{\kappa_{y}}\right)+\left[\mathbf{A}^{-1} \mathbf{C}\right]^{(1,2)}\left(\frac{\left.\partial H_{y}\right|_{\bar{k}} ^{n+1 / 2}}{\kappa_{z}}\right) \\
& +\left[\mathbf{A}^{-1} \mathbf{C}\right]^{1,3\rangle}\left(\frac{\left.\partial \tilde{H}_{x}\right|_{\tilde{k}} ^{n+1 / 2}}{\kappa_{z}}\right)_{i+1 / 2, j, k}+\left[\mathbf{A}^{-1} \mathbf{C}\right]^{1,4\rangle}\left(\frac{\left.\partial \tilde{H}_{z}\right|_{i} ^{n+1 / 2}}{\kappa_{x}}\right)_{i+1 / 2, j, k} \\
& +\left[\mathbf{A}^{-1} \mathbf{C}\right]^{1,5\rangle}\left(\frac{\left.\partial \tilde{H}_{y}\right|_{i} ^{n+1 / 2}}{\kappa_{x}}\right)_{i+1 / 2, j, k}+\left[\mathbf{A}^{-1} \mathbf{C}\right]^{1,6\rangle}\left(\frac{\left.\partial \tilde{H}_{x}\right|_{j} ^{n+1 / 2}}{\kappa_{y}}\right)_{i+1 / 2, j, k} \\
& \left.+\frac{\Delta t}{\varepsilon_{0}} f_{x y}\right)_{i+1 / 2, j, j}^{n+1 / 2}-\left.\frac{\Delta t}{\varepsilon_{0}} f_{x z}\right|_{i+1 / 2, j, k} ^{n+1 / 2} .
\end{aligned}
$$

However, this conventional PML implementation in anisotropic magnetized plasma may lead to diverging FDTD results, which will be shown in the next section.

\section{NUMERICAL EXAMPLES}

For simplicity, without loss of generality, the one-dimensional (1D) problem (along the $\mathrm{z}$-axis) is considered for analyzing the EM wave interaction with anisotropic magnetized plasma. An $x$-polarized differentiated Gaussian pulse is considered in the $1 \mathrm{D}$ region of the anisotropic magnetized plasma region with an arbitrary angle $\theta\left(0^{\circ}, 30^{\circ}, 60^{\circ}\right.$, and $\left.90^{\circ}\right)$ between the wave-
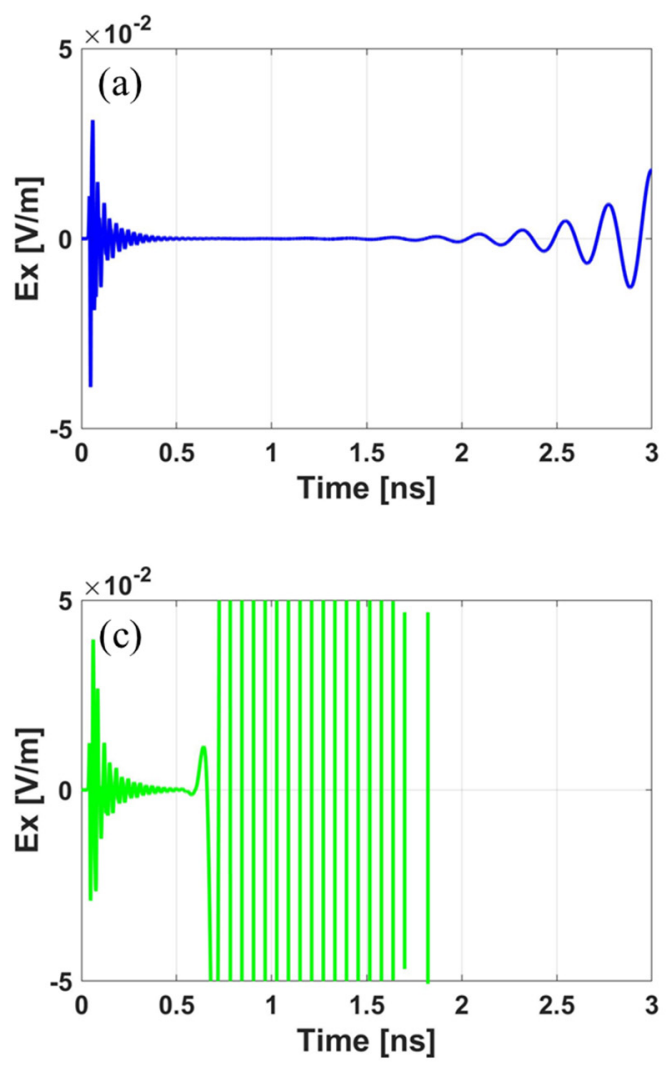

number vector and the DC bias magnetic field. The computational domain contains 500 cells with a uniform grid $\Delta z=75$ $\mu \mathrm{m}$ and a temporal cell size $\Delta t=0.2475$ ps. Ten cells of CFSPML were used at the terminations of the space to eliminate unwanted reflections. The plasma parameters are modeled to have an electron density without ions under an applied $1.7 \mathrm{~T}$ magnetic field. Therefore, the electron plasma frequency $\omega_{p}=$ $2 \pi \times 50 \times 10^{9} \mathrm{rad} / \mathrm{s}$, the electron cyclotron frequency $\omega_{b}=$ $3 \times 10^{11} \mathrm{rad} / \mathrm{s}$, and the electron collision frequency $v_{c}=20 \times 10^{9}$ $\mathrm{Hz}[18]$.

Let us consider the conventional PML implementation, e.g., (29). Fig. 1 shows the time-domain waveforms of the $E_{x}$ field component at the observation point located 50 cells away from the source. As shown in Fig. 1, the conventional PML-FDTD simulation results are inaccurate and even divergent in all cases.

Now, let us consider the proposed PML implementation, e.g., (22). Fig. 2 shows that the FDTD simulation results are numerically stable in this case. As shown in Fig. 2, the $E_{y}$ field component exists under the $x$-polarized incident field. This implies that the proposed FDTD algorithm can successfully analyze the anisotropic phenomenon of magnetized plasma.

We then conduct a parametric study for the proposed PML performance. An angle between the wavenumber vector and the DC bias magnetic field is $0^{\circ}$. All the other parameters are the same as in the previous simulation. The reference solution is
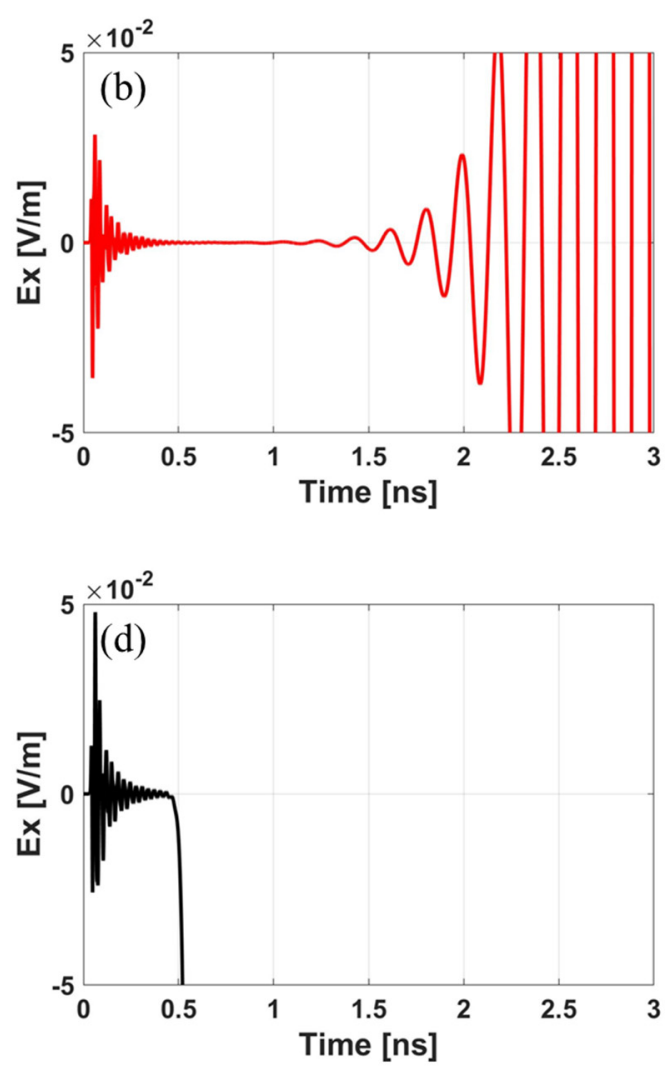

Fig. 1. Conventional PML implementation results of the $E_{x}$ field component in the anisotropic magnetized plasma region with an arbitrary angle $\theta$. (a) $\theta=0^{\circ}$, (b) $\theta=30^{\circ}$, (c) $\theta=60^{\circ}$, and (d) $\theta=90^{\circ}$. 


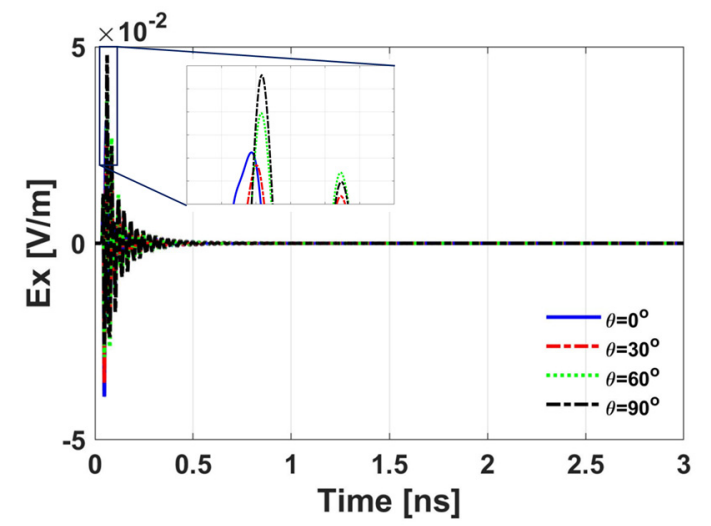

(a)

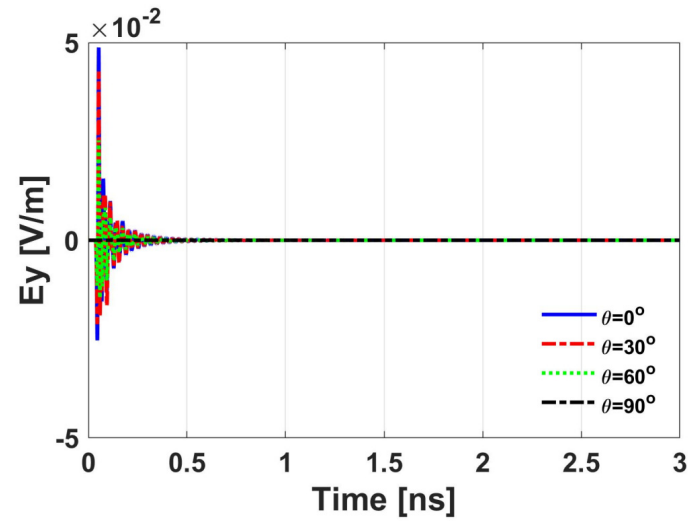

(b)

Fig. 2. Proposed PML implementation results of the electric field components in the anisotropic magnetized plasma region with an arbitrary angle $\theta$. (a) $E_{x}$ field and (b) $E_{y}$ field.

obtained using a large FDTD domain such that the reference solution is not contaminated by PML reflections [1]. The reflection error from the PML is defined as

$$
\text { Rel.error }(t)=20 \log _{10} \frac{\left|E(t)-E^{r e f}(t)\right|}{\max \left(\left|E^{r e f}(t)\right|\right)}
$$

where $E(t)$ is the proposed PML implemented electric field, and $E^{\text {ref }}(t)$ is the reference electric field.

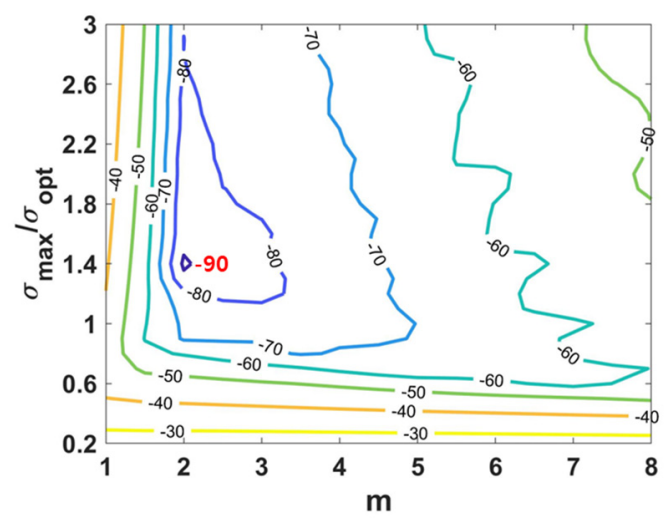

(a)
Here, $\sigma_{\text {opt }}$ is scaled as follows [1]:

$$
\sigma_{\mathrm{opt}}=\frac{0.8(m+1)}{\eta_{0} \Delta \zeta}
$$

where $\eta_{0}$ is the free-space wave impedance, and $\Delta$ is the latticecell dimension. As shown in Fig. 3, the optimum CFS factors are $m=2, \sigma_{\max } / \sigma_{\text {opt }}=1.4, \kappa_{\max }=2$, and $\alpha_{\max }=2$. These factors

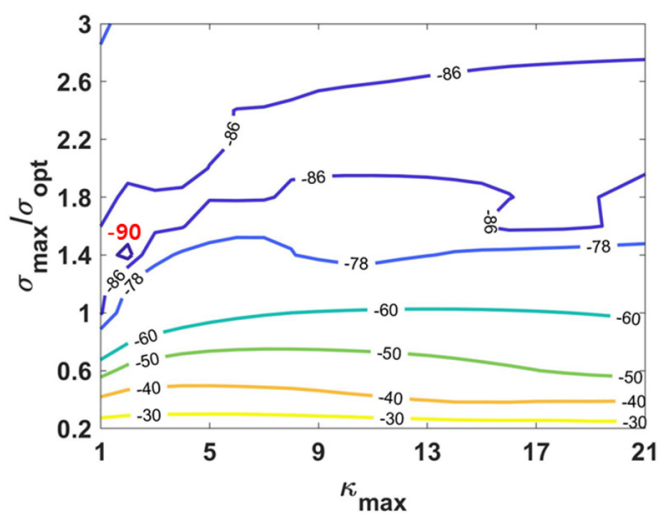

(b)

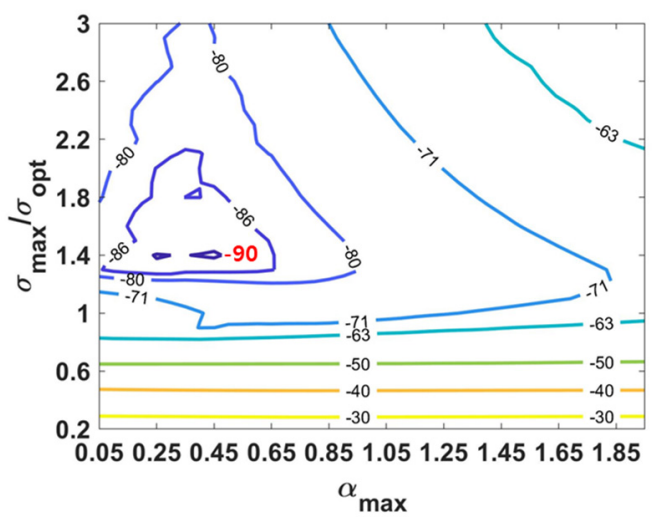

(c)

Fig. 3. Contour plots of the maximum reflection error as a function of the CFS factors. (a) $\kappa_{\max }=2, \alpha_{\max }=0.45$. (b) $m=2, \alpha_{\max }=0.45$. (c) $m=2$, $\kappa_{\max }=2$. 
yield the lowest reflection error of $-90 \mathrm{~dB}$. We also compute the relative error for the optimum CFS factors under various DC bias magnetic fields. Fig. 4 shows that the PML performance is good, and thus the proposed PML implementation is suitable for the EM analysis of anisotropic magnetized plasma.

Finally, we compute the right-hand circularly polarized (RCP) and left-hand circularly polarized (LCP) reflection coefficients of the EM wave in the magnetized plasma slab with an arbitrary angle $\theta\left(0^{\circ}, 30^{\circ}\right.$, and $\left.60^{\circ}\right)$. The computational domain is divided into 500 cells, and the plasma slab occupies 120 cells. All the other parameters are kept the same as in the previous simulation. As shown in Fig. 5, the proposed E-J PML-FDTD simulation results have a good agreement with the analytic results [19].

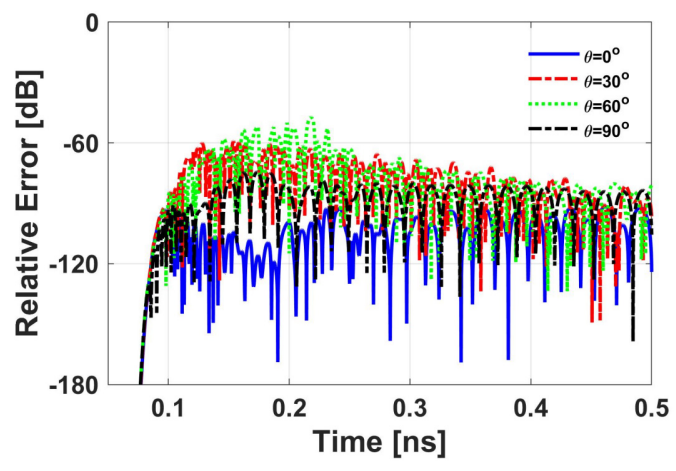

Fig. 4. Relative error of the CFS-PML E-J collocated FDTD.

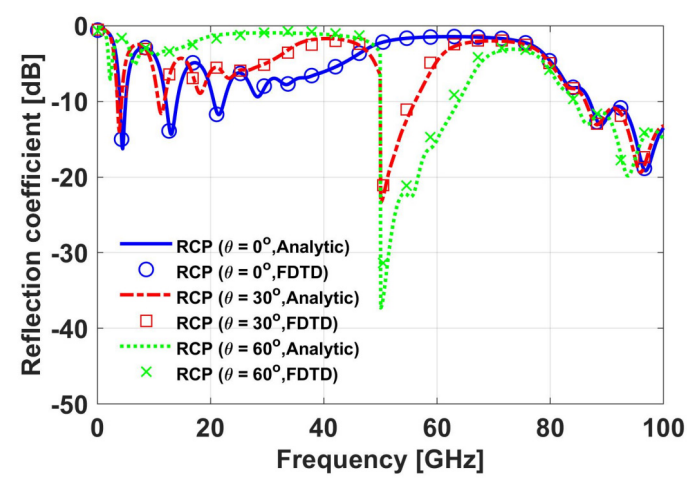

(a)

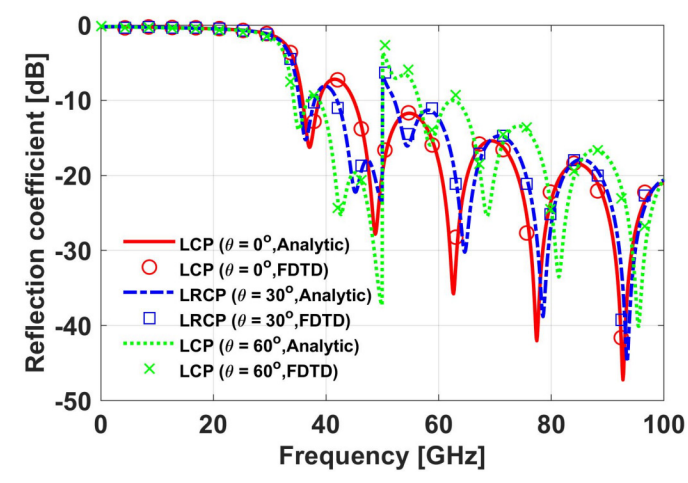

(b)

Fig. 5. Reflection coefficients: (a) RCP and (b) LCP.

\section{CONCLUSION}

In this work, we have proposed a stable PML implementation suitable for the accurate FDTD method for the analysis of EM wave propagation in anisotropic magnetized plasma. Toward this purpose, we have developed an accurate E-J collocated FDTD algorithm for the plasma region with an arbitrary geomagnetic field by including the PML variables in the FDTD matrix update equations. The proposed PML-FDTD method can be applied to accurately predict EM wave propagation in the atmosphere for radio and satellite communication.

This work was supported by the research fund of Signal Intelligence Research Center supervised by Defense Acquisition Program Administration and Agency for Defense Development of Korea.

\section{REFERENCES}

[1] A. Taflove and S. C. Hagness, Computational Electrodynamics: The Finite-Difference Time-Domain Method, 3rd ed. Norwood, MA: Artech House, 2005.

[2] R. Chilton, K. Y. Jung, R. Lee, and F. L. Teixeira, "Frozen modes in parallel-plate waveguides loaded with magnetic photonic crystals," IEEE Transactions on Microwave Theory and Techniques, vol. 55, no. 12, pp. 2631-2641, 2007.

[3] H. Chung, K. Y. Jung, X. T. Tee, and P. Bermel, "Time domain simulation of tandem silicon solar cells with optimal textured light trapping enabled by the quadratic complex rational function," Optics Express, vol. 22, no. S3, pp. A818A832, 2014.

[4] S. Park, S. Kim, D. K. Kim, J. Choi, and K. Y.Jung, "Numerical study on the feasibility of a $24 \mathrm{GHz}$ ISM-band Doppler radar antenna for near-field sensing of human respiration in electromagnetic aspects," Applied Sciences, vol. 10, no. 18, article no. 6159, 2020.

[5] J. L. Young, "A full finite difference time domain implementation for radio wave propagation in a plasma," Radio Science, vol. 29, no. 6, pp. 1513-1522, 1994.

[6] Y.Yu and J. Simpson, "An E-J collocated 3-D FDTD model of electromagnetic wave propagation in magnetized cold plasma," IEEE Transactions on Antennas and Propagation, vol. 58, no. 2, pp. 469-478, 2010.

[7] B. R. Mahafza, Radar Systems Analysis and Design using $M A T L A B, 2$ nd ed. Boca Raton, FL: CRC Press, 2002.

[8] F. Hunsberger, R. Luebbers, and K. Kunz, "Finite-difference time-domain analysis of gyrotropic media. I. Magnetized plasma," IEEE Transactions on Antennas and Propagation, vol. 40, no. 2, pp. 1489-1495, 1992. 
[9] D. M. Sullivan, "Z-transform theory and the FDTD method," IEEE Transactions on Antennas and Propagation, vol. 44, no. 1, pp. 28-34, 1996.

[10] T. Kashiwa and I. Fukai, "A treatment by FD-TD method of dispersive characteristics associated with electronic polarization," Microwave and Optical Technology Letters, vol. 3, no. 6, pp. 203-205, 1990.

[11] H. Choi, Y. H. Kim, J. W. Baek, and K. Y. Jung, "Accurate and efficient finite-difference time-domain simulation compared with CCPR model for complex dispersive media," IEEE Access, vol. 7, pp. 160498-160505, 2019.

[12] H. Choi, J. W. Baek, and K. Y.Jung, "Comprehensive study on numerical aspects of modified Lorentz model based dispersive FDTD formulations," IEEE Transactions on Antennas and Propagation, vol. 67, no. 12, pp. 7643-7648, 2019.

[13] Y. Fang, X. L. Xi, J. F. Liu, Y. R. Pu, and J. S. Zhang, "An iterative WLP-FDTD method for wave propagation in magnetized plasma," IEEE Transactions on Plasma Science, vol. 45, no. 8, pp. 2215-2219, 2017.

[14] J. Li and P. Wu, "Efficient PML implementation based on

Jeahoon Cho

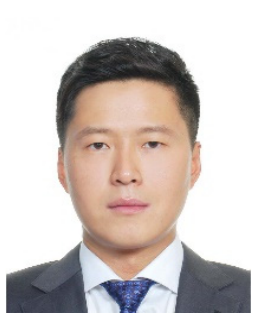

received his B.S. degree in communication engineering from Daejin University, Pocheon, Korea, and his M.S. and Ph.D. degrees in electronics and computer engineering from Hanyang University, Seoul, Korea, in 2004, 2006, and 2015, respectively. From 2015 to August 2016, he was a postdoctoral researcher at Hanyang University. Since September 2016, he has worked at Hanyang University, Seoul, Korea, where he is currently a research professor. His current research interests include computational electromagnetics and EMP/EMI/EMC analysis. the unconditionally stable CN-FDTD algorithm for anisotropic magnetized plasma," Optik, vol. 171, pp. 468-475, 2018.

[15] J. P. Berenger, "A perfectly matched layer for the absorption of electromagnetic waves," Journal of Computational Physics, vol. 114, no. 2, pp. 185-200, 1994.

[16] J. A. Roden and S. D. Gedney, "Convolutional PML (CPML): an efficient FDTD implementation of the CFSPML for arbitrary media," Microwave and Optical Technology Letters, vol. 27, pp. 334-339, 2000.

[17] K. Y. Jung, S. Ju, and F. L. Teixeira, "Application of the modal CFS-PML-FDTD to the analysis of magnetic photonic crystal waveguides," IEEE Microwave and Wireless Components Letters, vol. 21, no. 4, pp. 179-181, 2011.

[18] S. Liu, J. Mo, and N. Yuan, "Piecewise linear current density recursive convolution FDTD implementation for anisotropic magnetized plasmas," IEEE Microwave and Wireless Components Letters, vol. 14, no. 5, pp. 222-224, 2004.

[19] V. L. Ginzburg, The Propagation of Electromagnetic Waves in Plasmas, 2nd ed. New York, NY: Pergamon Press, 1970.

Kyung-Young Jung

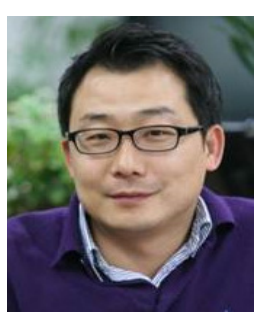

received his B.S. and M.S. degrees in electrical engineering from Hanyang University, Seoul, Korea, in 1996 and 1998, respectively, and his Ph.D. degree in electrical and computer engineering from The Ohio State University, Columbus, USA, in 2008. From 2008 to 2009 , he was a postdoctoral researcher at The Ohio State University, and from 2009 to 2010, he was an assistant professor at the Department of Electrical and Computer Engineering, Ajou University, Suwon, Korea. Since 2011, he has worked at Hanyang University, where he is now an associate professor at the Department of Electronic Engineering. His current research interests include computational electromagnetics, bioelectromagnetics, and nanoelectromagnetics.

\section{Min-Seok Park}

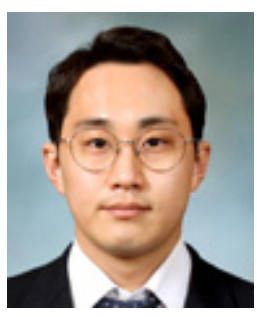

received his B.S. degree from the Department of Electrical Engineering at Myongi University, Yongin, Korea, in 2015, and his M.S. degree in electrical engineering from Hanyang University, Seoul, Korea, in 2017. From 2018 to 2020, he participated in EMTech's circuit and product development. He is currently pursuing his $\mathrm{Ph} . \mathrm{D}$. degree in electrical and computer engineering. His current research interests include computational electromagnetics, wave propagation, and multiphysics. 